\title{
Unilateral Oculomotor Nerve Palsy as an Initial Presentation of Bilateral Chronic Subdural Hematoma: Case Report
}

\author{
Ryosuke MATSUDA, ${ }^{1}$ Yasuo HIRONAKA, ${ }^{1}$ Hisashi KAWAI, ${ }^{1}$ Young-Su PARK, ${ }^{1}$ \\ Toshiaki TAOKA, ${ }^{2}$ and Hiroyuki NAKASE ${ }^{1}$
}

Departments of ${ }^{1}$ Neurosurgery and ${ }^{2}$ Radiology, Nara Medical University, Kashihara, Nara

\begin{abstract}
Isolated oculomotor nerve palsy is well known as a symptom of microvascular infarction and intracranial aneurysm, but unilateral oculomotor nerve palsy as an initial manifestation of chronic subdural hematoma (CSDH) is a rare clinical condition. We report a rare case of an 84-year-old woman with bilateral CSDH who presented with unilateral oculomotor nerve palsy as the initial symptom. The patient, who had a medical history of minor head injury 3 weeks prior, presented with left ptosis, diplopia, and vomiting. She had taken an antiplatelet drug for lacunar cerebral infarction. Computed tomography (CT) of the head showed bilateral CSDH with a slight midline shift to the left side. She underwent an urgent evacuation through bilateral frontal burr holes. Magnetic resonance angiography (MRA) after evacuation revealed no intracranial aneurysms, but constructive interference in steady-state (CISS) magnetic resonance imaging (MRI) revealed that the left posterior cerebral artery (PCA) ran much more anteriorly and inferiorly compared with the right PCA and the left oculomotor nerve passed very closely between the left PCA and the left superior cerebellar artery (SCA). There is the possibility that the strong compression to the left uncus, the left PCA, and the left SCA due to the bilateral CSDH resulted in left oculomotor nerve palsy with an initial manifestation without unconsciousness. Unilateral oculomotor nerve palsy as an initial presentation caused by bilateral CSDH without unconsciousness is a rare clinical condition, but this situation is very important as a differential diagnosis of unilateral oculomotor nerve palsy.
\end{abstract}

Key words: oculomotor nerve palsy, bilateral chronic subdural hematoma, posterior cerebral artery

\section{Introduction}

Chronic subdural hematoma (CSDH) normally occurs in the elderly after a mild head injury. ${ }^{11} \mathrm{CSDH}$ is a common condition, especially among patients who take antiplatelet and anticoagulant drugs after coronary heart disease and cerebral infarction. ${ }^{1)}$ Most patients with CSDH present with gait disturbance, hemiparesis, headache, dementia, urinary incontinence, and consciousness disturbance. ${ }^{5)}$

On the other hand, the most common cause of isolated oculomotor nerve palsy is microvascular infarction which is caused as a result of diseases, such as diabetes mellitus, hypertension, atherosclerosis, and collagen vascular disease. ${ }^{8}$ Another common cause of isolated oculomotor nerve palsy is direct compression by an intracranial aneurysm, usually an internal carotid-posterior communicating artery aneurysm that sometimes presents a pupil-sparing oculomotor nerve palsy as an early symptom of enlarging aneurysm. ${ }^{97}$

Received September 28, 2012; Accepted December 26, 2012
To date, only 3 cases of CSDH presenting with unilateral oculomotor nerve palsy as an initial manifestation have been reported in conscious patients in the English literature $^{2,4,8)}$ We review these cases and discuss the mechanism of unilateral oculomotor nerve palsy associated with CSDH.

\section{Case Report}

An 84-year-old woman with a history of lacunar infarction, who was undergoing treatment with an antiplatelet drug, suffered from occipital head injury and went to the local hospital. Computed tomography (CT) of the head revealed a left thin acute subdural hematoma in the left frontal convex with no cranial fractures (Fig. 1). She had no neurological deficits and went home on that day. Three weeks later, she returned to the local hospital presenting with the left ptosis, diplopia, and vomiting. CT of the head revealed bilateral CSDH that was predominant on the right side (Fig. 2). She was eventually transferred to our hospital for further treatment. Her Glasgow Coma 


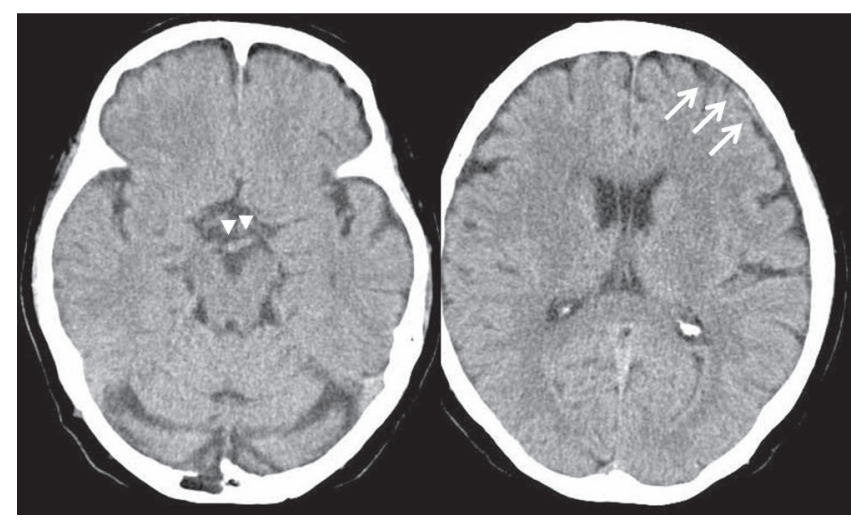

Fig. 1 Computed tomography (CT) showing a left thin acute subdural hematoma in the frontal convex (arrows) and a calcification in the basilar artery and left posterior cerebral artery (arrow heads).

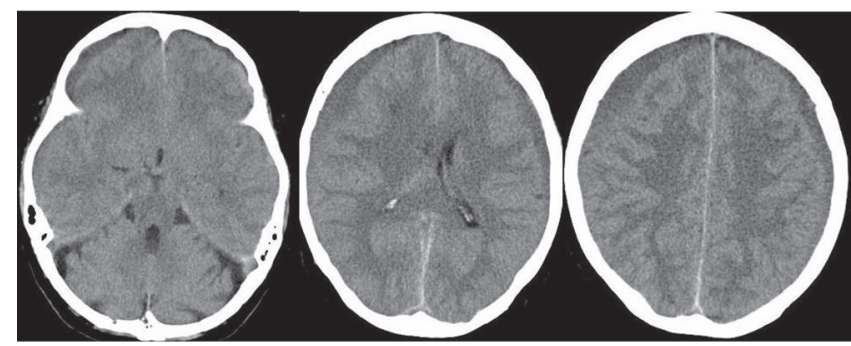

Fig. 2 Computed tomography (CT) showing the bilateral chronic subdural hematoma with a slight midline shift to the left side 3 weeks later after the initial mild head injury.

Scale was 15. Ophthalmologic examination showed left complete oculomotor nerve palsy with left ptosis, diplopia, and dilated pupil. She underwent an urgent evacuation through bilateral frontal burr holes under local anesthesia. After bilateral burr hole surgery, we initially opened the outer membrane of the subdural hematoma on the right side, and immediately opened the outer membrane on the left side to avoid worsening the uncal herniation. The pressures of the hematoma were very high bilaterally. We inserted the drains bilaterally into the cavity of the hematoma.

The drains were removed on the day after the surgery. Her ptosis and diplopia resolved immediately after the surgery, and her dilated left pupil was improving gradually but still remained at the time of discharge. Magnetic resonance angiography (MRA) revealed mild stenosis of the left internal carotid artery in the cavernous portion, but no intracranial aneurysms (Fig. 3). Magnetic resonance imaging (MRI) revealed no abnormal findings surrounding the brainstem. However, constructive interference in steadystate (CISS) MRI revealed that the P1 and P2 portions of the left posterior cerebral artery (PCA) ran much more
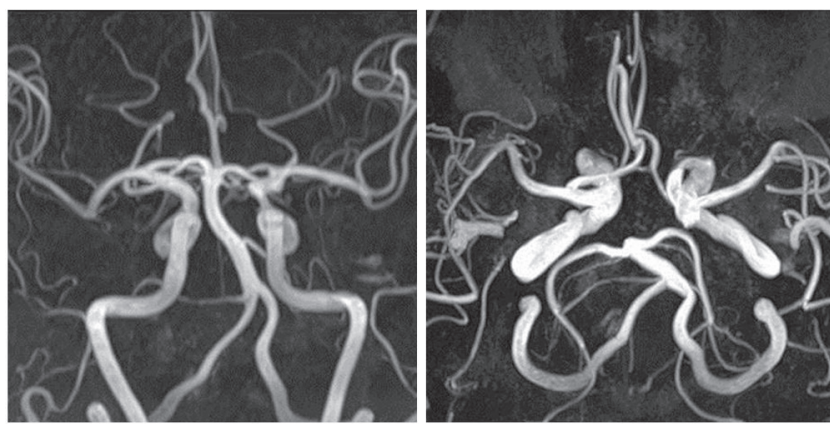

Fig. 3 Postoperative magnetic resonance angiography (MRA) showing mild stenosis of the left internal carotid artery in the cavernous portion, but no intracranial aneurysms (left), and demonstrating that the left posterior cerebral artery runs much more anteriorly (right).

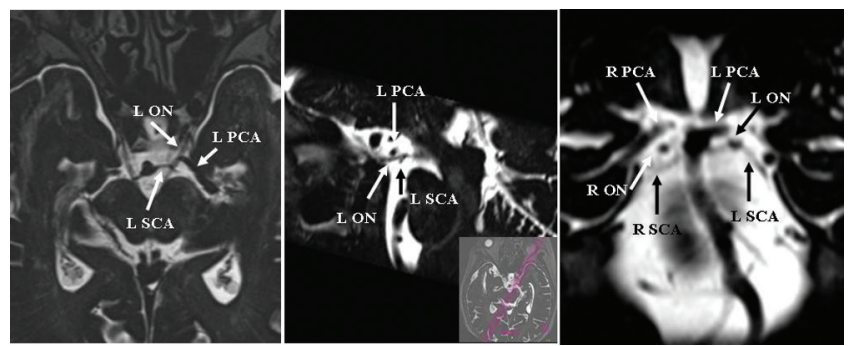

Fig. 4 Postoperative constructive interference in steady-state (CISS) magnetic resonance (MR) imaging revealing that the P1 and P2 portions of the left posterior cerebral artery (PCA) ran much more anteriorly above the left oculomotor nerve (ON) (left). Oblique-sagittal CISS MR imaging revealed that the left ON was very near to the PCA and superior cerebellar artery (SCA), inset: scout view (center). Coronal CISS MR imaging revealing the wide space surrounding the right $\mathrm{ON}$, but the tight space surrounding the left ON (right).

anteriorly compared to the right PCA. Moreover, it is suspected that the strong compression to the left uncus, the left PCA and the left superior cerebellar artery (SCA) due to bilateral CSDH would result in the left complete oculomotor nerve palsy (Fig. 4). The patient subsequently resumed her normal life without any neurological deficits, except for the anisocoria. The patient has been observed for 6 months and though she recovered from the ptosis and diplopia her anisocoria remains. Repeated CT revealed no recurrence of CSDH.

\section{Discussion}

To date, only 3 cases of CSDH initially presenting with unilateral oculomotor nerve palsy without coma have been reported in the English literature. A summary of CSDH with initial presentation of oculomotor nerve palsy 
Table 1 Summary of chronic subdural hematoma with an initial presentation of oculomotor nerve palsy without unconsciousness

\begin{tabular}{lccccccc}
\hline $\begin{array}{l}\text { Author } \\
\text { (years) }\end{array}$ & Age/sex & $\begin{array}{c}\text { Complication } \\
\text { (drugs) }\end{array}$ & Symptom & $\begin{array}{c}\text { Type of oculomotor } \\
\text { nerve palsy }\end{array}$ & $\begin{array}{c}\text { Side of } \\
\text { hematoma }\end{array}$ & $\begin{array}{c}\text { Type of } \\
\text { operation }\end{array}$ & $\begin{array}{c}\text { Postoperative results of } \\
\text { oculomotor nerve palsy }\end{array}$ \\
\hline $\begin{array}{l}\text { Crone } \\
(1985)\end{array}$ & 66/F & Diabetes mellitus & $\begin{array}{c}\text { Rt. oculomotor nerve } \\
\text { palsy H/A }\end{array}$ & Pupil-sparing & Rt & Craniotomy & Complete recovery \\
1 month later
\end{tabular}

F: female, H/A: headache, HT: hypertension, Lt.: left, M: male, NA: not available, RT: right, TIA: transient ischemic attack.

without coma is shown in Table $1 .^{2,4,8)}$ Among these 4 cases, including our case, 2 patients were women and 1 was a man and the other was not known. The mean age was 70 years old (range: 60-84 years). Two patients took anti-coagulants; one was warfarin, the other was aspirin. One patient had a history of diabetes mellitus. Preoperative radiological findings showed bilateral CSDH in 3 cases and unilateral CSDH in 1 case. One patient presented a pupil-sparing oculomotor nerve palsy and the others presented complete oculomotor nerve palsy. In this series, the oculomotor nerve palsy improved between 1 week and 3 months after surgery. There was no mention of the relationship between the oculomotor nerve and the PCA.

\section{Etiology of oculomotor nerve palsy}

In general, unilateral oculomotor nerve palsy with unconsciousness is one of the most important symptoms of cerebral herniation. In particular, if the patients present with unconsciousness, ipsilateral oculomotor nerve palsy, and contralateral hemiparesis, after severe traumatic head injury and cerebral infarction, then urgent treatment of decompressive surgery should be considered. ${ }^{3,11)}$ On the other hand, the most common cause of isolated oculomotor nerve palsy in a conscious subject is microvascular infarction caused by diseases, such as diabetes mellitus, hypertension, atherosclerosis, or collagen vascular disease. ${ }^{8)}$ Another common cause of isolated oculomotor nerve palsy is direct compression by an intracranial aneurysm; usually an internal carotid-posterior communicating artery aneurysm. Especially, a pupil-sparing oculomotor nerve palsy is known as an early symptom of enlarging aneurysm, because anatomically parasympathetic fibers supplying pupillary functions pass along the dorsal medial surface of oculomotor nerve, so are preserved to compress by internal carotid-posterior communicating artery aneurysms projecting laterally from the carotid artery. ${ }^{9)}$ While it is a rare condition, some case reports have revealed that direct compression of the oculomotor nerve by the PCA induced complete oculomotor nerve palsy and microvascular decompression were useful in such cases. ${ }^{6,10)}$

\section{Mechanism of oculomotor nerve palsy due to CSDH}

In this case, although the subdural hematoma was predominant on the right side, left complete oculomotor nerve palsy occurred. This discrepancy could be explained as follows: A slight midline shift to the left side and subsequent compression of the upper brainstem were induced gradually by the right subdural hematoma. On the other hand, postoperative CISS MRI demonstrated that the P1 and P2 portions of the left PCA ran much more anteriorly and inferiorly over the left oculomotor nerve compared to the right PCA and that the left oculomotor nerve was very close to both the left PCA and the SCA in the cisternal segment. We suspected that the left oculomotor nerve was compressed by the left PCA and the left SCA when the left uncus was dislocated medially due to the left CSDH combined with the co-existing compression to the upper brainstem from the right subdural hematoma. This mechanism could explain why the left oculomotor nerve palsy occurred alone. After evacuation of the bilateral hematoma, the symptom of left oculomotor nerve palsy started to improve immediately. We considered that this immediate recovery from the oculomotor nerve palsy except pupil dysfunction after the surgery further supported this mechanism.

As mentioned in the previous paragraph, bilateral CSDH takes an important role in the oculomotor nerve palsy. In the literature, bilateral CSDH may present rare central nerve disorders including divergence palsy as the result of subsequent compression of the upper brainstem. ${ }^{7)}$ In fact, 3 of the 4 cases of CSDH with isolated oculomotor nerve palsy presented bilateral CSDH.

CSDH with unilateral oculomotor nerve palsy without unconsciousness is a rare condition but an important differential diagnosis. In such cases, we considered that CT of the head is very useful to diagnose CSDH. If the patient presented the isolated oculomotor nerve palsy due to CSDH, we recommended an urgent evacuation of 
the CSDH to avoid progressive aggravation and to recover from the oculomotor nerve palsy.

\section{Conflicts of Interest Disclosure}

We have no conflicts about this case report.

\section{References}

1) Asghar M, Adhiyaman V, Greenway MW, Bhowmick BK, Bates A: Chronic subdural haematoma in the elderly-a North Wales experience. J R Soc Med 95: 290-292, 2002

2) Crone KR, Lee KS, Davis CH: Oculomotor palsy with pupillary sparing in a patient with chronic subdural hematoma. Surg Neurol 24: 668-670, 1985

3) Delashaw JB, Broaddus WC, Kassell NF, Haley EC, Pendleton GA, Vollmer DG, Maggio WW, Grady MS: Treatment of right hemispheric cerebral infarction by hemicraniectomy. Stroke 21: 874-881, 1990

4) Kurokawa Y, Ishizaki E, Inaba K: Incomplete oculomotor nerve palsy caused by an unruptured internal carotidanterior choroidal artery aneurysm-case report-. Neuro Med Chir (Tokyo) 45: 143-147, 2005

5) Mori K, Maeda M: Surgical treatment of chronic subdural hematoma in 500 consecutive cases: clinical characteristics, surgical outcome, complications, and recurrence rate. Neurol Med Chir (Tokyo) 41: 371-381, 2001

6) Nakagawa H, Nakajima S, Nakajima Y, Furuta Y, Nishi O,
Nishi K: Bilateral oculomotor nerve palsies due to posterior cerebral arterial compression relieved by microvascular decompression-case report. Neurol Med Chir (Tokyo) 31: 45-48, 1991

7) Okudera H, Saito J, Matsuo T: A case of divergence palsy associated with bilateral chronic subdural hematoma. No To Shinkei 43: 183-186, 1991

8) Phookan G, Cameron M: Bilateral chronic subdural haematoma: an unusual presentation with isolated oculomotor nerve palsy. J Neurol Neurosurg Psychiatry 57: 1146, 1994

9) Saito R, Sugawara T, Mikawa S, Fukuda T, Kohama M, Seki H: Pupil-sparing oculomotor nerve paresis as an early symptom of unruptured internal carotid-posterior communicating artery aneurysms-three case reports. Neuro Med Chir (Tokyo) 48: 304-306, 2008

10) Suzuki K, Muroi A, Kujiraoka Y, Takano S, Matsumura A: Oculomotor palsy treated by microvascular decompression. Surg Neurol 70: 210-212, 2008

11) Tseng SH: Reduction of herniated temporal lobe in patients with severe head injury and uncal herniation. J Formos Med Assoc 91: 24-28, 1992

Address reprint requests to: Ryosuke Matsuda, MD, PhD, Department of Neurosurgery, Nara Medical University, 840 Shijo-cho, Kashihara, Nara 634-8522, Japan. e-mail: cak93500@pop02.odn.ne.jp 